\title{
Animal dispersal modelling: Handling landscape features and related animal choices
}

\author{
Séverine Vuilleumier*, Richard Metzger \\ Institute of Environmental Science and Technology, Swiss Federal Institute of Technology of Lausanne, \\ CH-1015 Lausanne, Switzerland
}

Received 13 October 2004; received in revised form 11 April 2005; accepted 15 April 2005

Available online 9 June 2005

\begin{abstract}
Animal dispersal in a fragmented landscape depends on the complex interaction between landscape structure and animal behavior. To better understand how individuals disperse, it is important to explicitly represent the properties of organisms and the landscape in which they move. A common approach to modelling dispersal includes representing the landscape as a grid of equal sized cells and then simulating individual movement as a correlated random walk. This approach uses a priori scale of resolution, which limits the representation of all landscape features and how different dispersal abilities are modelled.

We develop a vector-based landscape model coupled with an object-oriented model for animal dispersal. In this spatially explicit dispersal model, landscape features are defined based on their geographic and thematic properties and dispersal is modelled through consideration of an organism's behavior, movement rules and searching strategies (such as visual cues). We present the model's underlying concepts, its ability to adequately represent landscape features and provide simulation of dispersal according to different dispersal abilities. We demonstrate the potential of the model by simulating two virtual species in a real Swiss landscape. This illustrates the model's ability to simulate complex dispersal processes and provides information about dispersal such as colonization probability and spatial distribution of the organism's path.
\end{abstract}

(c) 2005 Elsevier B.V. All rights reserved.

Keywords: Landscape model; Animal movement; Dispersal behavior modelling; Landscape feature; Object-oriented design; Spatially explicit model

\footnotetext{
* Corresponding author at: The Ecology Centre, The University of Queensland, St. Lucia, Qld 4072, Australia. Tel.: +61 73365 1686; fax: +61733651655.

E-mail addresses: severine.vuilleumier@epfl.ch (S. Vuilleumier), richard.metzger@epfl.ch (R. Metzger).
}

\section{Introduction}

Understanding how animals disperse is a major issue for the management and conservation of fragmented populations. Landscape heterogeneity and fragmentation affects how organisms are distributed in the landscape (Fahrig and Merriam, 1985; Turner, 1989; Kennedy and Gray, 1997). It determines the 
chance of a patch being colonized (Henein and Merriam, 1990; Hansson, 1991; Taylor et al., 1993; Hanski, 1999; Hanski and Ovaskainen, 2000), can reduce inbreeding in small populations and maintains evolutionary potential (Barton, 1992; Driscoll, 1998; Couvet, 2002). To understand dispersal, it is important to not only consider the dispersal capabilities of the organism but also the complex interaction between the organism's behavior and landscape pattern and use. This is widely recognized in the ecological literature but seldom considered explicitly (Reed and Dobson, 1993; Curio, 1996; Lima and Zollner, 1996; Ulfstrand, 1996; Sutherland, 1998; Caro, 1999; Reed, 1999; Anthony and Blumstein, 2000).

Modelling of animal dispersal provides a useful paradigm for investigating these complex interactions and is seen as an essential conceptual tool for landscape conservation planning (Kareiva and Wennergren, 1995; King and With, 2002; Kramer-Schadt et al., 2004). Due to the difficulty in gathering and interpreting experimental results on animal dispersal processes, simulation models have become a cost-effective approach to understanding dispersal dynamics (Koenig et al., 1996; Tischendorf, 1997; Wiegand et al., 1999; Pretsler et al., 2000; Tischendorf and Fahrig, 2000; Brillinger et al., 2002). Simulation models with spatially explicit landscapes enable the integration of the relationships between species and the landscape and provide representation of the spatial elements that promote or constrain dispersal. Several dispersal models with spatially explicit landscapes have been developed. Some consider dispersal behavior according to habitat affinity or physiological states in order to assess animal movements or provide guidelines for landscape and wildlife management (With and Crist, 1995; Gustafson and Gardner, 1996; Lindenmayer and Possingham, 1996; Blackwell, 1997; With et al., 1997, 1999; Farnsworth and Beecham, 1999; Thulke et al., 1999; Bergman et al., 2000; Wu et al., 2000; Gardner and Gustafson, 2004).

At present, two main kinds of landscape models grid and patch, are used to model spatially structured populations in a continuous landscape (Hanski and Simberloff, 1997; Bian, 2003). These models are characterized by two types of data structures: grid-based and vector-based data structures. Network-based models (Zollner and Lima, 1999b; Conradt et al., 2003) are another approach to modelling landscape with particular emphasis to modelling suitable habitat. We do not consider these models in this paper, as they do not have a continuous representation of the landscape.

\subsection{Grid-based models}

In dispersal modelling the spatial representation of a landscape is commonly based on grid models where the landscape is represented by a finite number of equally sized cells (Burrough and McDonnell, 1998; With and Crist, 1995; With et al., 1997; Beecham and Farnsworth, 1998; Tischendorf et al., 1998; Carter and Finn, 1999; Thulke et al., 1999; Bergman et al., 2000; Wissel, 2000; Gardner and Gustafson, 2004). These cells can be squares, triangles, hexagons or any other shape that can be used to tessellate the 2D plane. Each cell contains one or more values, which represent attributes of the landscape such as vegetation types, elevation, and temperature. Discretizing the landscape in this way enables flexibility in spatial analysis and mathematical modelling (Burrough and McDonnell, 1998). It offers simple and efficient methods for incorporating state transitions based on properties of a cell and its neighboring cells as is used in cellular automaton (With and King, 1997; Thulke et al., 1999; Wissel, 2000; Anderson and Neuhauser, 2002; Chen et al., 2002).

The criticisms of grid-based models cover three main lines of argument: (i) the existence of an a priori fixed scale of resolution, (ii) in some cases attributes of cells will need to be aggregated (usually an average) at the pre-defined scale and (iii) the limitation in representing line features and topology (shape and relationships between distant objects) (Laurini and Thompson, 1992). The resolution of grid-based models requires a trade off between landscape representation and dispersal mechanisms. If a grid-based approach is used to represent narrow linear features like roads and rivers accurately then the grid will need to be at a very fine scale. This fine resolution may not be appropriate for larger landscape features, such as forests, as it may not capture all the properties of the feature. For landscape features represented by multiple grid cells, parameters associated with the entire landscape feature are distributed into fixed resolution cells instead of having one value being assigned to the entire landscape feature. Conversely, with increased cell size, linear and point landscape features cannot be represented with sufficient accuracy. If a large cell is adopted then the cells that contain linear features, such as roads or streams, 
will have their properties averaged over the entire cell and will not be accurately represented. For example at a cell size of a hectare then a road will never be much more than $10 \%$ of any cell. To accurately represent the road, we would need to have grid cell of 1/100th of a hectare.

Grid-based models also have limitations for modelling dispersal as the grid cell resolution for both landscape and individual movement are identical (Tischendorf, 1997). That is, the step-time dispersal distance and the organism's perceptual range are defined by the scale at which the landscape is modelled. In these types of models, individuals have to move across adjacent cells in predefined directions. Modelling the movement of individuals that use linear structures (i.e. hedges or rivers) is usually not feasible, as these features are generally not adequately represented at the chosen resolution of the entire map. In addition, the extent of the study area, the number of simulated individuals, and the ability to carry out a sensitivity analysis are limited due to large computational times and memory capacity required to simulate dispersal using grid-based models (Tischendorf, 1997; Koenig et al., 1996). Thus, we believe grid-based modelling approaches do not provide an effective universal framework for modelling all dispersal traits.

\subsection{Vector-based models}

Vector data structures represent landscape features according to their shapes and functions via points, polylines, and polygons associated with multiple geographic and non-geographic attributes (Burrough and McDonnell, 1998; Bian, 2003). In vector-based models the topological properties of objects (i.e. shapes, neighbors and hierarchy), and the relationships between objects can be described explicitly. Links between objects can be related to their position or their typology (i.e. all forest patches may be related to each other). Although less common than grid models, vector models are particularly well adapted to modelling landscape features and are a useful method for investigating dispersal processes (Bian, 2003). Vector-based models provide further developmental opportunities in animal movement simulations such as movement along linear network features or between stepping stone habitats. They also allow animal choice during dispersal such as animal attraction to specific resources, patch configuration preferences or avoidance of human infrastructures.

The added functionality and flexibility of vectorbased models comes with costs (Tischendorf, 1997; Bian, 2003). Data handling is complex and computationally demanding. For instance, each time a spatial object is changed or created, the topological relationships of the neighboring objects need to be updated. Vector-based models require sophisticated database management, which may incur a learning curve and subsequent cost. Vector models are limited in representing gradients of spatially continuous variables (i.e. environmental factors), since object attributes are spatial homogeneous. Despite these technical issues, vector-based models are well suited for modelling landscapes and investigating dispersal dynamics, as they are able to incorporate the geometry of the patch networks and spatial relationships between landscape features.

\subsection{Object-oriented dispersal modelling}

The simulation of dispersal in a fragmented landscape may be dramatically improved by using an object-oriented approach (Rumbaugh et al., 1991; Downing and Reed, 1996; Beecham and Farnsworth, 1998; Lorek and Sonnenschein, 1999). This allows an explicit representation of individuals, the heterogeneous landscape and the interactions between them (Downing and Reed, 1996; Tischendorf, 1997; Ziv, 1998; Lomnicki, 1999; Bian, 2000; Bian, 2003). Objects, such as landscape features and dispersers, have properties and behaviors depending on their attributes and behavioral values which define their spatial, geometric, temporal and thematic dimensions (Rumbaugh et al., 1991; Worboys, 1995). As information is shared between objects, it allows the simulation of dynamic processes through changes in state or behavior. Combining the available information on and between objects with dispersal movement rules, allows creation of chains of spatial objects. These chains are themselves objects and as such, have their own properties. For instance, chains may represent individual travel paths, and properties represent dispersal distance and dispersal time.

Few studies have used an explicit landscape model coupled with an object-oriented modeling approach. Among them, Beecham and Farnsworth (1998) and 
Carter and Finn (1999) developed a grid-based landscape model coupled with an object-oriented dispersal model to investigate animal foraging behavior. These studies were based on grid models, which are limited in how the landscape can be represented and how dispersal is modeled. No studies have incorporated the objected-oriented dispersal model with a vector-based landscape model.

We present a spatially explicit dispersal model that includes a detailed representation of landscape structures and permits the simulation of individual movements between landscape features according to dispersal ability and behavior. To do this we develop a vector-based landscape model coupled with an object-oriented model for animal dispersal where landscape features are defined based on their geographic and thematic properties. The model has the ability to incorporate dispersal abilities through consideration of behavioral and physiological traits of individuals and allows animals to move according to a variety of movement paradigms. Animals can move within the entire landscape, or along linear network features, such as roads or rivers. We illustrate the utility and potential of this method through examples.

\section{Methods}

We develop the idea of representing a landscape as a mesh of irregular spatial objects. This is illustrated for a basic vector-data model covering an area of Switzerland. Then we describe the spatially explicit dispersal model, which selects appropriate landscape features and informs dispersing individuals about their environment.

\subsection{Conceptual design}

The landscape representation is made up of a mesh of spatial objects (vector-based). These objects may be irregular in shape and dimension, but are homogeneous in regard to their properties. The landscape structure is fully described by two kinds of objects: patches and frontiers. A patch is a homogeneous area that represents land cover and its related properties. Patches are limited by frontiers, which are transitions between patches of different land cover and linear features, such as roads, hedges or rivers. Both patches and frontiers contain all (a)

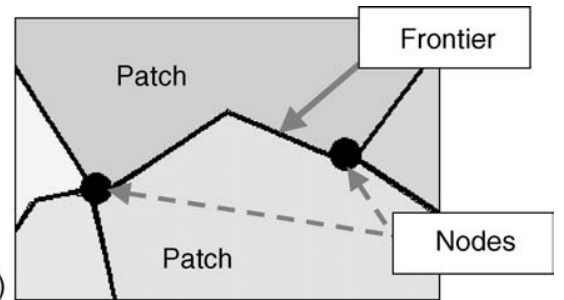

(b)

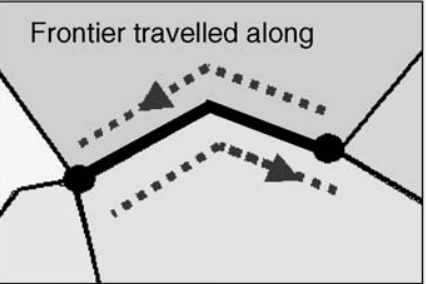

(c)

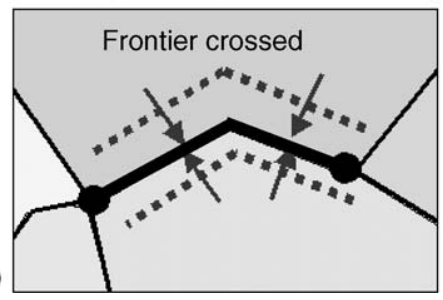

Fig. 1. Representation of landscape structure including patches (shaded areas), frontiers (black lines) and nodes (black circles) (a). Frontiers allow movement along (b) and across (c) in both directions.

the spatial and thematic related information associated with the feature it represents.

Patches are connected to their surrounding frontiers while the frontiers are connected to each other through nodes (Fig. 1). This allows the simulation of different kinds of movements and landscape uses. Animals can use either linear features or polygons during dispersal. Organisms disperse from one patch to the next via the frontiers. To enable a rich variety of animal movements, frontiers are designed to allow movement in each direction along the frontier and movement across the frontier (Fig. 1).

Two important parameters in modelling animal dispersal are perceptual range and dispersal distance (Kozakiewicz and Szacki, 1995; Koenig et al., 1996; Lima and Zollner, 1996; Bowman et al., 2002). The perceptual range is the distance from which a particular landscape element (i.e. a habitat patch) can be perceived by a given animal (Lima and Zollner, 1996; Zollner and Lima, 1999a; Zollner, 2000). Perceptual range is modeled by creating a buffer in which an 
animal can perceive the target object. Dispersal distance depends on the landscape structure, the paths used, and the dispersal abilities of the animal species (Bell, 1991). To model dispersal distance, metrics were added as attributes to patches and frontiers. For a patch, the distance attribute is the measured distance between two surrounding frontiers. One frontier separates the current patch and the previous patch and the second frontier separates the current patch and the next patch. The distance is measured from the middle points of each frontier. This provides a good approximation of the distance between patches even if patches are long and thin. Important biases appear when patches have arc-like shapes. These patches are identified by a line drawn between two frontiers in a patch. If this line intercepts more than a threshold number of patches, then the patch is split into two separate patches. This threshold depends on the species and landscape under consideration. In the case of frontiers, the distance attribute is the length of the frontier. Animals can disperse through some landscape features more easily than others. To account for this cost, dispersal distance is either weighted according to the landscape features suitability or as additional time spent in a landscape feature.

\subsection{The landscape model construction}

The landscape model was developed based on basic vector data. The Swiss Federal Office of Topography (SwissTopo) provided data covering the whole of Switzerland at a scale of 1:25,000 (Gurtner, 1997). The landscape is represented by a complete network of mutually exclusive polygons, overlaid by points and several networks of polylines. Polygons represent land cover, points are isolated landscape features such as a tree or a cave; polylines are networks such as hedges, or road, rail and hydrographical networks.

The transformation of the vector data, into patches, frontiers and nodes is achieved using MapBasic ${ }^{\circledR}$, scripts within the MapInfo Professional ${ }^{\circledR}$ environment. This involves a number of steps including data integrity analysis, patches creation, frontier creation, and the calculation of perceptual distance.

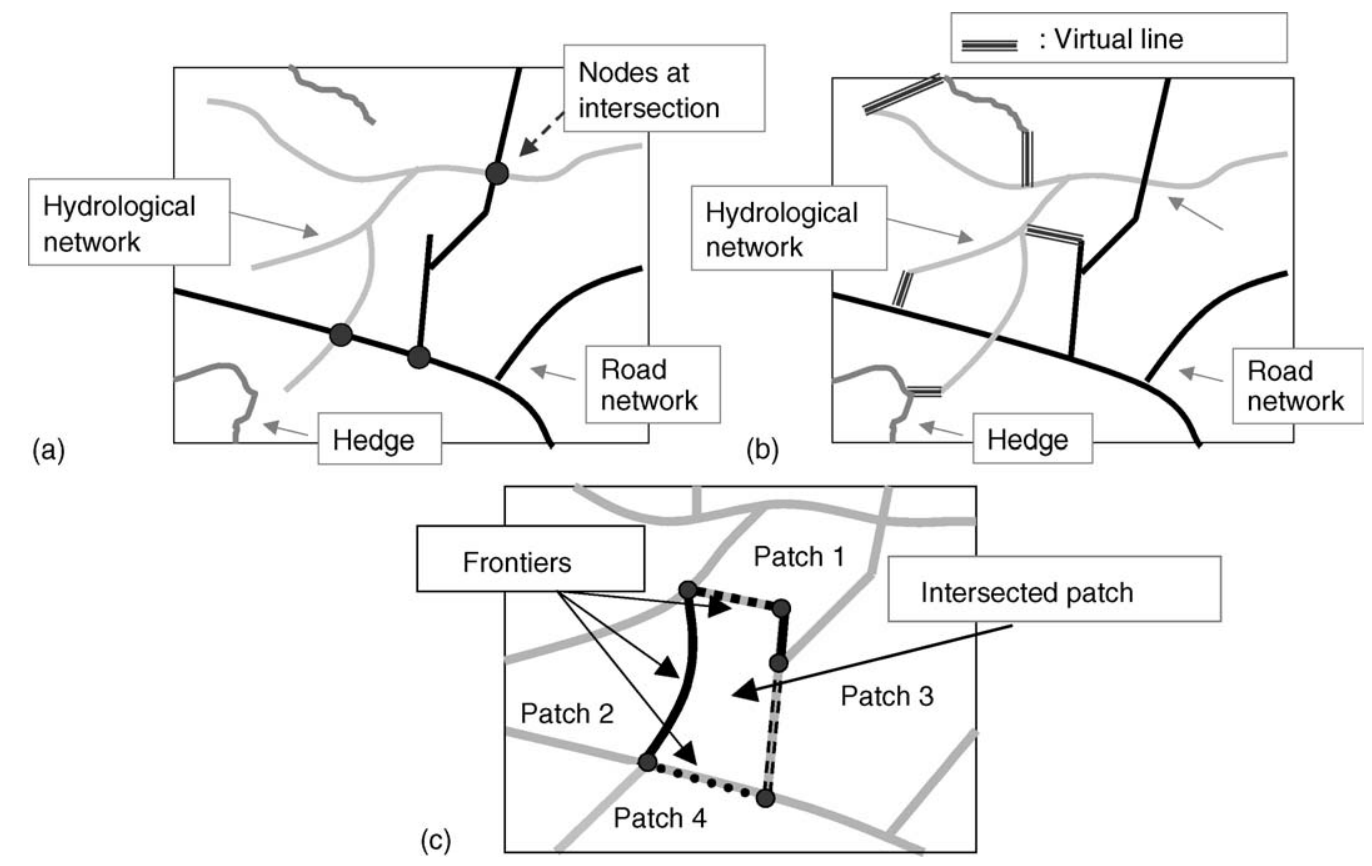

Fig. 2. Polygon and polyline creation. Polyline networks (hedges, and road and hydrological networks) are connected by adding a common node at their intersection (a). Virtual lines (thick striped line) are added to linear network in order to form closed polylines (b). Frontiers (black solid and dashed lines) are intersections between adjacent patches. This example illustrates 5 unique frontiers for the active patch (c). 
Data integrity analysis. Data are checked and corrected if topological problems such as multiple points, overlaps or self-intersections are detected.

Patch creation. All polyline networks are overlaid and at each intersection point, the related polylines are split and a node is added (Fig. 2a). The resulting elements are gathered in a single layer to form a clean polyline network. If a polyline is not closed, as may be the case for a starting point of a river, an algorithm creates a 'virtual' line, with no associated attributes, and joins it to the nearest neighboring polyline (Fig. 2b) creating a complete network of closed polylines. The polygons of the land cover layer are then split with the new polyline layer resulting in a complete irregular network of patches matching the polyline network.

Frontier creation. Frontiers are created by intersecting adjacent patches. They are identified using three tests (Fig. 2c). Firstly, each neighboring patch must have a non-null common frontier, the second verifies that the patch and its neighbor do not share the same attributes (to avoid selection of itself) and the third test checks that the entire frontier belongs to both adjacent patches. An algorithm adds the appropriate landscape feature attributes to the created frontier by comparing its position with the initial polyline networks.

Calculation of perceptual distance. The perceptual distance is modeled as a buffer zone from a 'target object' that intersects patches and frontiers. A 'target object' may represent suitable habitat, a resource, or a breeding site. The target object and the distance from it are added to each intersected patch and frontier as attributes.

\subsection{Spatially explicit dispersal model}

To simulate complex interactions between individuals and the landscape, we coupled the landscape model with a dispersal model. This is developed in an objectoriented programming (OOP) environment using Borland $^{\circledR}$ Delphi ${ }^{\mathrm{TM}}$. Individuals and the landscape

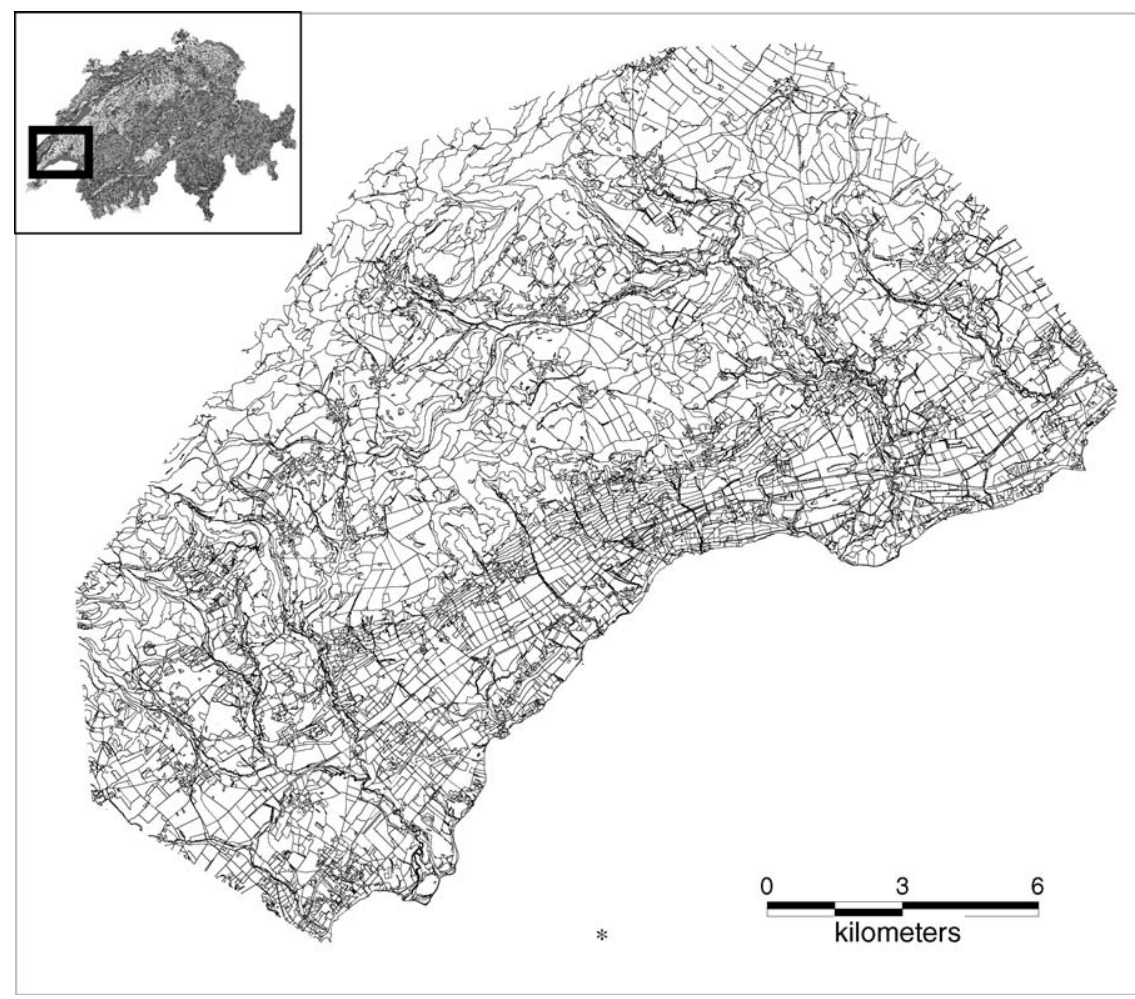

Fig. 3. The pattern of the feature-oriented landscape model after discretization into patches and frontiers. Each patch represents a unique continuous landscape feature. 
features are represented as objects. Objects belong to linked lists (i.e. chaining, Sedgewick (1988)), which are themselves objects (e.g. lists of landscape features such as rivers or forest). These lists encapsulate information about spatial and non-spatial relationships with other objects. Information is shared between objects and can be dynamic. Objects can also be updated as a response to changes in the state of other objects.

Simulation of animal dispersal benefits from the communication between objects. Animals disperse based on movement rules. These rules depend on the behavioral traits of the modeled species, their response to landscape features, and their ability to perceive their environment. In the movement process, the choice of successive spatial objects is based on their attributes, such as the shape, and their ability to be crossed, as well as the species-specific movement rules. The sequence of chosen objects during dispersal forms a chain that defines the individual's path. These paths contain all the information related to the spatial objects they contain. The combination of patches, frontiers and nodes allows the simulation of several kinds of movements. In particular, this model allows the movement of individuals according to linear landscape features, which can be simulated by a selection of suitable frontiers. This type of movement may be appropriate for modelling the dispersal of small mammals (Peles et al., 1999).

Three inputs are required to initiate the building of an individual's path: (i) the landscape model, (ii) the starting habitat patches, and (iii) the individual and its specific behavior and state, which includes dispersal distance, the dispersal objective, the animal movement rules, and the animal preferences. For instance the dispersal objective may be to find a new habitat patch. A simulation begins at the starting habitat patch, defined by the user, which initiates the path and becomes the active object. According to user-defined animal movement rules, a selection is made among the objects linked to the active object, resulting in a list of suitable objects. Animal movement rules can be related to the animal's ability to move, such as fight, swim or move through or over obstacles such as fences. Choosing procedures corresponding to the species behavior leads to the selection of the next object becoming active. The choosing procedure can be related to the patches or/and frontiers attributes of such shape, suitability, attractiveness or occupancy. Once an object has been selected, it is added to the path, distance covered is updated and all object attributes are recorded including the number of times it has been used. These selections and choosing procedure are repeated until the individual reaches the objective or exceeds its dispersal abilities (i.e. maximum dispersal distance). Model output includes information about the dispersal path, including dispersal success, distance covered, and frequency of landscape use. The model simulates thousands of individuals enabling the calculation of colonization probabilities, the distribution of dispersal distances, and the pattern of corridors between two habitat patches.

\subsection{Model applications}

We illustrate the potential of the dispersal model through an example. A landscape model is built for a fragmented area in western Switzerland covering $260 \mathrm{~km}^{2}$ (Fig. 3). It represents a complex network of
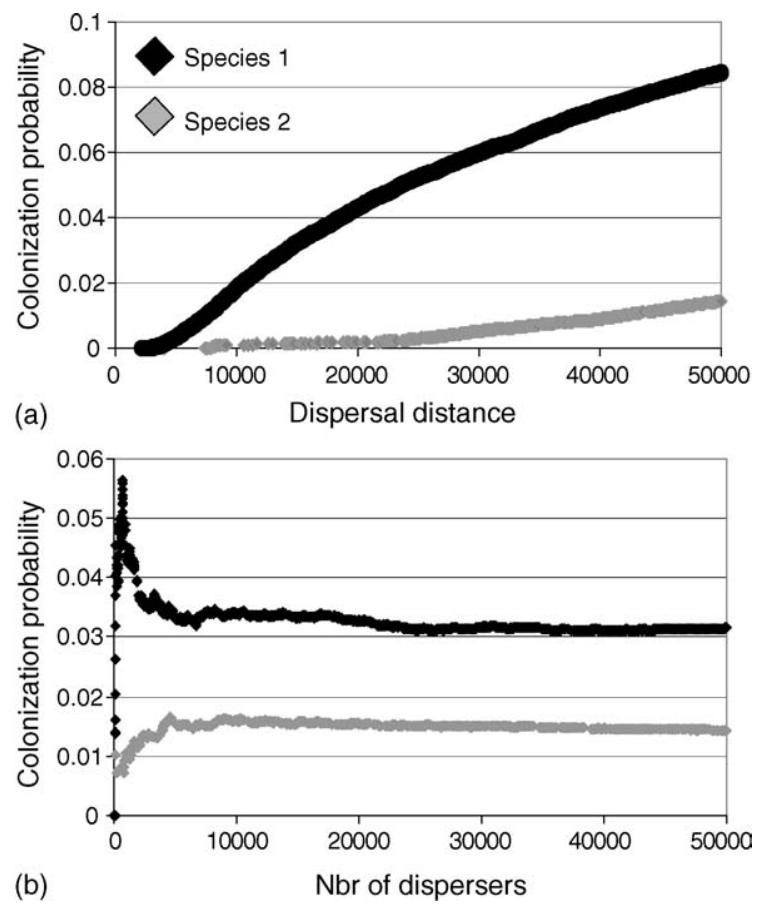

Fig. 4. Two examples of output from the dispersal model for the two virtual species-species 1: ungulate and species 2: small mammal-including probability of colonization as a function of assigned dispersal distance (a) and as a function of the number of dispersers (b). 
patches and frontiers that are irregular in shape and dimension and reflect the true landscape features. In this landscape we simulate dispersal for two species with different dispersal capabilities. Factors such as the number of individuals released, the relationship between dispersal distance and colonization probability, and spatial path are analyzed.

Dispersal is simulated for two "virtual species", firstly for a species that closely resembles the dispersal capabilities and behavior of an ungulate and secondly of a small mammal. Dispersal starts from an initial habitat patch. The objective for an individual is to find a new habitat patch. For the ungulate, individuals are assumed to use the whole landscape, i.e. move from patch to patch through frontiers. They are assumed to perceive and be attracted by a habitat patch within $100 \mathrm{~m}$. The transition probability of movement from patch $i$ to patch $j$ via frontier, $i j$, is computed dynamically and depends on the position and the attractiveness of a patch in relation to other patches around it. If a habitat patch is within $100 \mathrm{~m}$ then the individual will perceive it and travel there by the shortest route. If a habitat patch is not within $100 \mathrm{~m}$ then a transition probability is assigned to each frontier $i j$. This probability depends on the relative attractiveness of the frontier and the adjacent cell. The attractiveness is defined by expert judgment according to the degree of naturalness. For instance the attractiveness of a highway may be very low and assigned a value of 0 , while for a forest, the attractiveness may be high and assigned a value of 0.8 . Once an individual has left a patch it is not permitted to return to the patch in the next time step, unless the individual is trapped (i.e. all other frontier transition probabilities are zero). Transition probabilities of the frontiers in the patch are normalized in order to sum to one, and a pseudorandom number is used to determine which frontier and corresponding patch is selected. Unlike the ungulate, for the small mammal, individuals are assumed to only move along the linear landscape features. During dispersal at each node, the next linear feature is selected at random. For both species, a dispersal event is successful if an individual reaches a new habitat patch without exceeding the dispersal distance assigned. Each time a landscape feature is used, the number of time used is incremented and the path followed by each individual (succession of patches or frontiers) is recorded.

The model provides useful outputs for analyzing dispersal processes in a fragmented landscape. It allows the fluxes of individual movements between

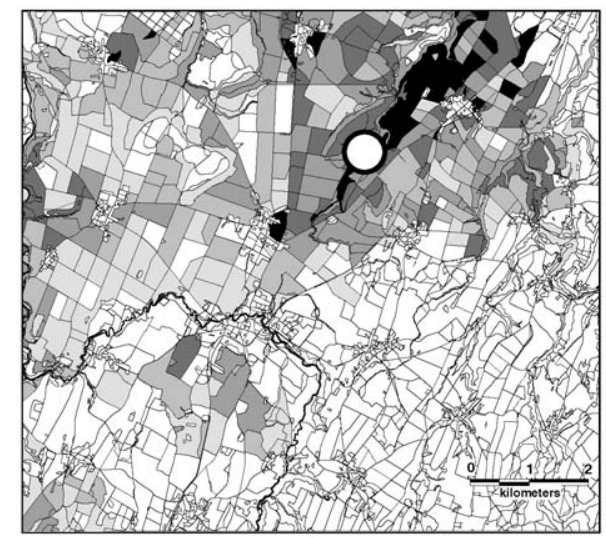

Number of patches being crossed Starting point

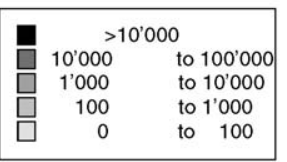

(a)

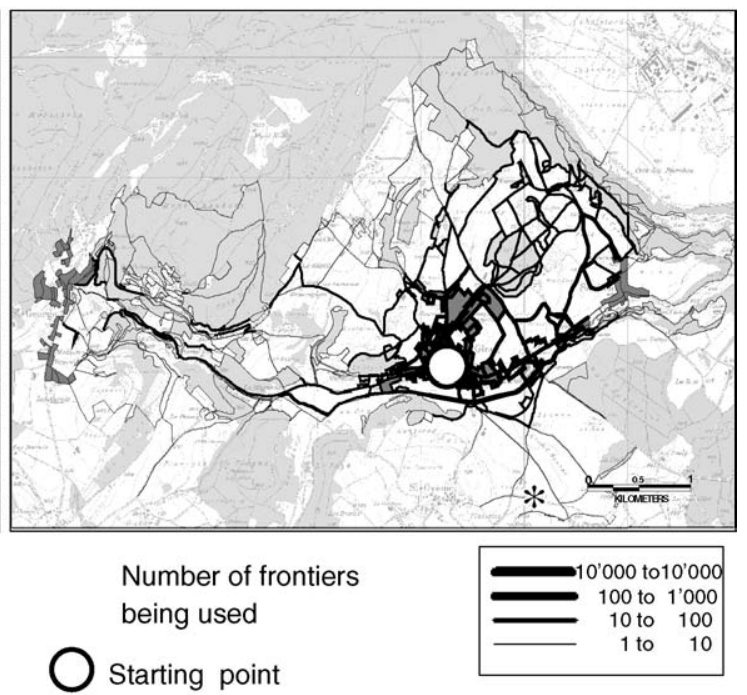

(b)

Fig. 5. Patterns of the frequency of landscape use generated by simulations of 50,000 for the two virtual species dispersing from a starting point either using all landscape features chosen with an assigned probability (maximum dispersal distance is $50 \mathrm{~km}$ ) (a) or using only the linear features of the landscape chosen randomly (maximum dispersal distance is $15 \mathrm{~km})(\mathrm{b})$. 
habitat patches to be quantified. Since path characteristics, such as distance covered, number and characteristics of patches used are tracked, the calculation of colonization probability as a function of dispersal distance is possible (Fig. 4a). It also enables the estimation of how many dispersers are necessary for a constant probability of colonization. Here simulations suggest that in order to accurately estimate colonization probabilities the number of dispersers must be greater than 20,000 (simulation times $<10 \mathrm{~min}$ on a $1.8 \mathrm{GHz}$ processor pc) (Fig. 4b). This was evident for both dispersal types. The pattern of landscape use for the two species illustrates the number of times landscape objects are used by successful individuals (Fig. 5). This kind of representation is ideal for analyzing ecological networks and corridors.

\section{Discussion and conclusion}

In recent years, the increase in computer performance has permitted the development of complex animal movement simulation models. Since experimental tracking and analyses of the movement behavior of individuals remains a time consuming, expensive and difficult challenge (Koenig et al., 1996; Tischendorf, 1997), modelling provides a bridge between the results of experimental studies and the information needed for critical management decisions (Tischendorf et al., 1998).

Simulating ecological processes provide a powerful tool from both a theoretical and an applied point of view (Tischendorf et al., 1998; Berger et al., 1999; Grimm et al., 1999; Halle and Halle, 1999; Tyre et al., 2001). Spatial modelling of animal dispersal may be useful when evaluating the consequences of changing landscape structures on dispersal pattern (Gustafson and Gardner, 1996; With et al., 1999). They are particularly useful for exploring alternative management strategies for species living in increasingly fragmented habitats (Lindenmayer and Possingham, 1996) and for developing management tools for the control of diseases (Thulke et al., 1999).

True dispersal processes implicitly integrate landscape complexity and dispersal behavior. Unfortunately in high fragmented landscapes grid-based modelling approaches may be too simple to simulate them explicitly. The spatially explicit dispersal model presented here is an innovative modelling framework enabling explicit mapping of the landscape, where linear structures are heavily present (Fig. 3). This is often poorly represented in currently used raster-based landscape models even though they are quite significant in dispersal processes. The discretization into patches and frontiers respects the criss-cross pattern of a real landscape and therefore conserves patches shapes and related properties. These attributes can be used, if necessary, as parameters for dispersal modelling.

While the discretization of the landscape is limited by the complexity of data handling and the need of a sophisticated database management, the advantages of this approach far outweigh the limitations. While raster-based models require a trade off between landscape representation and dispersal mechanisms when choosing an appropriate grid size, this approach is not constrained to a particular resolution. Thus small elements like hedges may be included in the model as well as large areas such as continuous forest. Different values can be used for patch size, dispersal movement and perceptual range. This is not the case in raster-based systems where the perceptual range must correspond to one or more cell sizes (e.g. With and Crist, 1995; Gustafson and Gardner, 1996; With et al., 1997, 1999; Farnsworth and Beecham, 1999; Bergman et al., 2000; King and With, 2002).

By representing landscape features as objects within a vector-based system, each landscape feature can contain the necessary attributes, providing information for simulating complex animal dispersal processes. Information can be related to land cover type, resource availability, shape attributes as well as carrying capacity, number of individuals and the presence of competitors or predators. The vector-based data structure enables the simulation of numerous dispersal events within larger landscapes, reducing biases between dispersal distance and the size of the study area (Tischendorf, 1997; Bian, 2003).

The implementation of the landscape model in an object-oriented programming framework provides flexibility in simulating dispersal processes. Complex and dynamic interactions can be simulated by assigning objects properties, behavior, and relationships (Beecham and Farnsworth, 1998; Carter and Finn, 1999). Coupling vector-based landscape models with 
object-oriented programming offers great improvements to modeling individual interactions with the landscape. One major advantage is the ability to dynamically update attributes during the simulation resulting a representation more realistic of the dispersal events. The patch selection is also computed dynamically. It can depend on the previous step in dispersal, the position and arrangement of the patches in the landscape as well as the proximity of a habitat patches. Such dynamic patch selection is not provided by current dispersal modelling approaches (Gustafson and Gardner, 1996; Beecham and Farnsworth, 1998; King and With, 2002).

The model allows the simulation of different kinds of movements such as along linear features, stepping stone or the movement across the entire landscape mosaic. As with other recent models (e.g. With et al., 1999; King and With, 2002; Gardner and Gustafson, 2004) it also provides the means for analyzing the spatial distribution of animal pathways during dispersal and a quantification of the colonization success based on movement constraints.

The model offers an ideal tool for further analyses of the effect of factors such as behavior, social structure, competition and predation on dispersal processes. Modelling within an object-oriented paradigm (Lorek and Sonnenschein, 1998; Ziv, 1998; Lorek and Sonnenschein, 1999; Ginot et al., 2002; Bousquet and Le Page, 2004) allows the integration of factors which may be important during dispersal such as animal learning, dispersal risk, life history, spacing behavior and conspecific attractions (Hestbeck, 1982; Smith and Peacock, 1990; Koenig et al., 1996; Lima and Zollner, 1996; Wolff, 1997; Wolff, 1999). Moreover, since changing scale is not a problem, simulating animal dispersal at the metapopulation scale (flux between populations) and at a local scale (corridor planning, local management plan) is possible. This model offers a new tool that will improve our understanding of the interactions between landscape structures and population ecology.

\section{Acknowledgements}

We thank three anonymous reviewers and Tracey Regan for their comments on previous versions of the manuscript.

\section{References}

Anderson, K., Neuhauser, C., 2002. Patterns in spatial simulations-are they real? Ecol. Model. 155, 19-30.

Anthony, L.L., Blumstein, D.T., 2000. Integrating behaviour into wildlife conservation: the multiple ways that behaviour can reduce N-e. Biol. Conserv. 95, 303-315.

Barton, N.H., 1992. The genetic consequences of dispersal. In: Stenseth, N.C., Lidicker, W.Z.J. (Eds.), Animal Dispersal: Small Mammals as a Model. Chapman and Hall, London, pp. 37-59.

Beecham, J.A., Farnsworth, K.D., 1998. Animal foraging from an individual perspective: an object oriented model. Ecol. Model. 113:, 141-156.

Bell, W.J., 1991. Searching Behaviour: The Behavioural Ecology of Finding Resources. Chapman and Hall, London, 358 pp.

Berger, U., Wagner, G., Wolff, W.F., 1999. Virtual biologist observes virtual grasshoppers: an assessment of different mobility parameters for the analysis of movement patterns. Ecol. Model. 115, 119-127.

Bergman, C.M., Schaefer, J.A., Luttich, S.N., 2000. Caribou movement as a correlated random walk. Oecologia 123, 364-374.

Bian, L., 2000. Component modeling for the spatial representation of wildlife movements. J. Environ. Manage. 59, 235-245.

Bian, L., 2003. The representation of the environment in the context of individual-based modeling. Ecol. Model. 159, 279-296.

Blackwell, P.G., 1997. Random diffusion models for animal movement. Ecol. Model. 100, 87-102.

Bowman, J., Jaeger, J.A.G., Fahrig, L., 2002. Dispersal distance of mammals is proportional to home range size. Ecology 83, 2049-2055.

Brillinger, D.R., Preisler, H.K., Ager, A.A., Kie, J.G., 2002. The Use of Potential Functions in Modelling Animal Movement. A festschrift honoring D.A.S. Fraser. pp. 369-386.

Bousquet, F., Le Page, C., 2004. Multi-agent simulations and ecosystem management: a review. Ecol. Model. 176, 313-332.

Burrough, A., McDonnell, A., 1998. Principles of Geographical Information Systems. Oxford University Press Inc., New York, $333 \mathrm{pp}$.

Caro, T., 1999. The behaviour-conservation interface. TREE 14, 366-369.

Carter, J., Finn, J.T., 1999. MOAB: a spatially explicit, individualbased expert system for creating animal foraging models. Ecol. Model. 119, 29-41.

Chen, Q., Mynett, A.E., Minns, A.W., 2002. Application of cellular automata to modelling competitive growths of two underwater species Chara aspera and Potamogeton pectinatus in Lake Veluwe. Ecol. Model. 147, 253-265.

Conradt, L., Zollner, P.A., Roper, T.J., Frank, K., Thomas, C.D., 2003. Foray search: an effective systematic dispersal strategy in fragmented landscapes. Am. Nat. 161, 905-915.

Couvet, D., 2002. Deleterious effects of restricted gene flow in fragmented populations. Cons. Biol. 16, 369-376.

Curio, E., 1996. Conservation needs ethology. TREE 11, 260263.

Downing, K., Reed, M., 1996. Object-oriented migration modelling for biological impact assessment. Ecol. Model. 93, 203-219. 
Driscoll, D.A., 1998. Genetic structure, metapopulation processes and evolution influence the conservation strategies for two endangered frog species. Biol. Cons. 83, 43-54.

Fahrig, L., Merriam, G., 1985. Habitat patch connectivity and population survival. Ecology 66, 1762-1768.

Farnsworth, K.D., Beecham, J.A., 1999. How do grazers achieve their distribution? A continuum of models from random diffusion to the ideal free distribution using biased random walks. Am. Nat. $153,509-526$.

Gardner, R.H., Gustafson, E.J., 2004. Simulating dispersal of reintroduced species within heterogeneous landscapes. Ecol. Model. 171, 339-358.

Ginot, V., Le Page, C., Souissi, S., 2002. A multi-agents architecture to enhance end-user individual-based modelling. Ecol. Model. $157,23-41$.

Grimm, V., Wyszomirski, T., Aikman, D., Uchmanski, J., 1999. Individual-based modelling and ecological theory: synthesis of a workshop. Ecol. Model. 115, 275-282.

Gurtner, M., 1997. Lecture de Carte: Manuel des Cartes Nationales. Club Alpin Suisse and Office fédéral de topographie, Wabern, Berne, 283 pp.

Gustafson, E.J., Gardner, R.H., 1996. The effect of landscape heterogeneity on the probability of patch colonization. Ecology 77, 94-107.

Halle, S., Halle, B., 1999. Modelling activity synchronization in freeranging microtine rodent. Ecol. Model. 115, 165-176.

Hanski, I., 1999. Metapopulation Ecology. Oxford University Press, Oxford, $313 \mathrm{pp}$.

Hanski, I., Simberloff, D., 1997. The metapopulation approach, its history conceptual domain and application to conservation. In: Hanski, I.A., Gilpin, M.E. (Eds.), Metapopulation Biology: Ecology, Genetics, and Evolution. Academic Press, San Diego, pp. $5-26$.

Hanski, I., Ovaskainen, O, 2000. The metapopulation capacity of a fragmented landscape. Nature 404, 755-758.

Hansson, L., 1991. Dispersal and connectivity in metapopulations. Biol. J. Lin. Soc. 42, 89-103.

Henein, K., Merriam, G., 1990. The element of connectivity where corridor quality is variable. Landsc. Ecol. 4, 157-170.

Hestbeck, J.B., 1982. Population regulation of cyclic mammals-the social fence hypothesis. Oikos 32, 157-163.

Kareiva, P., Wennergren, U., 1995. Connecting landscape patterns to ecosystem and population processes. Nature 373, 299-302.

Kennedy, M., Gray, R.D., 1997. Habitat choice, habitat matching and the effect of travel distance. Behaviour 134, 905-920.

King, A.W., With, K.A., 2002. Dispersal success on spatially structured landscapes: when do dispersal pattern and dispersal behavior really matter? Ecol. Model. 147, 23-39.

Koenig, W.D., Van Vuren, D., Hooge, P.N., 1996. Detectability, philoparty, and the distribution of dispersal distances in vertebrates. TREE 11, 514-517.

Kozakiewicz, M., Szacki, J., 1995. Movements of small mammals in a landscape: patch restriction or nomadism? In: Lidicker, W.Z. (Ed.), Landscape Approaches in Mammalian Ecology and Conservation, Minneapolis, Minesota, pp. 78-94.

Kramer-Schadt, S., Revilla, E., Wiegand, T., Breitenmoser, U., 2004. Fragmented landscapes, road mortality and patch connectivity: modelling dispersal for the Eurasian lynx in Germany. J. Appl. Ecol. 41, 711-723.

Laurini, R., Thompson, D., 1992. Fundamentals of Spatial Information Systems. Aademic Press Inc., London, 680 pp.

Lima, S.L., Zollner, P.A., 1996. Towards a behavioral ecology of ecological landscapes. TREE 11, 131-135.

Lindenmayer, D., Possingham, H., 1996. Ranking conservation and timber management options for Leadbetter's possum in SouthEastern Australia using population viability analysis. Cons. Biol. 10, 235-251.

Lomnicki, A, 1999. Individual-based models and the individualbased approach to population ecology. Ecol. Model. 115, 191-198.

Lorek, H., Sonnenschein, M., 1998. Object-oriented support for modelling and simulation of individual-oriented ecological models. Ecol. Model. 108, 77-96.

Lorek, H., Sonnenschein, M., 1999. Modelling and simulation software to support individual-based ecological modelling. Ecol. Model. 115, 199-216.

Peles, J.D., Bowne, D.R., Barrett, G.W., 1999. Influence of landscape structure on movement patterns of small mammals. In: Barrett, G.W., Peles, J.D. (Eds.), Landscape Ecology of Small Mammals. Springer-Verlag, New York, pp. 41-62.

Pretsler, H.K., Brillinger, D.R., Ager, A.A., Kie, J.G., 2000. Analysis of animal movement using telemetry and GIS data. Proc. Am. Stat. Ass., 100-105.

Reed, J.M., 1999. The role of behavior in recent avian extinctions and endangerments. Cons. Biol. 13, 232-241.

Reed, J.M., Dobson, A.P., 1993. Behavioral constraints and conservation biology—conspecific attraction and recruitment. TREE 8 , 253-256.

Rumbaugh, J., Blaha, M., Premerlani, M., Eddy, W., Lorensen, W., 1991. Object-orientation modeling and design, New York, 580 pp.

Sedgewick, R., 1988. Algorithms. Addison-Wesley, 657 pp.

Smith, A.T., Peacock, M.M., 1990. Conspecific attraction and the determination of metapopulation colonization rates. Cons. Biol. 4, 320-323.

Sutherland, W.J., 1998. The importance of behavioural studies in conservation biology. Anim. Behav. 56, 801-809.

Taylor, P.D., et al., 1993. Connectivity is a vital element of landscape structure. Oikos 68, 571-572.

Thulke, H.-H., Grimm, V., Müller, M.S., Staubach, C., Tischendorf, L., Wissel, C., Jeltsch, F., 1999. From pattern to practice: a scaling-down strategy for spatially explicit modelling illustrated by the spread and control of rabies. Ecol. Model. 117.

Tischendorf, L, 1997. Modelling individual movements in heterogeneous landscapes: potentials of a new approach. Ecol. Model. 103, 33-42.

Tischendorf, L., Fahrig, L., 2000. How should we measure landscape connectivity? Landsc. Ecol. 15, 633-641.

Tischendorf, L., Irmler, U., Hingst, R., 1998. A simulation experiment on the potential of hedgerows as movement corridors for forest carabids. Ecol. Model. 106, 107-118.

Turner, M.G., 1989. Landscape ecology: the effect of pattern on process. Annu. Rev. Ecol. Syst. 20, 171-197. 
Tyre, A.J., Possingham, H.P., Lindenmayer, D.B., 2001. Inferring process from pattern: can territory occupancy provide information about life history parameters. Ecol. Appl. 11, 1722-1737.

Ulfstrand, S., 1996. Behavioural ecology and conservation biology. Oikos 77, 183.

Wiegand, T., Moloney, K., Naves, J., Knauer, F., 1999. Finding the missing link between landscape structure and population dynamics: a spatially explicit perspective. Am. Nat. 154, 605-627.

Wissel, C., 2000. Grid-based Models as tools for Ecological Research. In: Dickmann, U., Law, R., Metz, J.A.J. (Eds.), The Geometry of Ecological Interactions Simplifying Spatial Complexity. Cambridge University Press, Cambridge, pp. 94-115.

With, K.A., Cadaret, S.J., Davis, C., 1999. Movement responses to patch structure in experimental fractal landscapes. Ecology 80, 1340-1353.

With, K.A., Crist, T.O., 1995. Critical thresholds in species' responses to landscape structure. Ecology 76, 2446-2459.

With, K.A., Gardner, R.H., Turner, M.G., 1997. Landscape connectivity and population distributions in heterogeneous environments. Oikos 78, 151-169.

With, K.A., King, A.W., 1997. The use and misuse of neutral landscape models in ecology. Oikos 79, 219-229.
Wolff, J.O., 1997. Population regulation in mammals: an evolutionary perspective. J. Anim. Ecol. 66, 1-13.

Wolff, J.O., 1999. Behavioral model systems. In: Barrett, G.W., Peles, J.D. (Eds.), Landscape Ecology of Small Mammals. Springer-Verlag, New York, pp. 11-40.

Worboys, M.F., 1995. GIS-A Computing Perspective. Taylor and Francis, London, 392 pp.

Wu, H., Li, B., Springer, T.A., Neill, W.H., 2000. Modelling animal movement as a persistent random walk in two dimensions: expected magnitude of net displacement. Ecol. Model. 132, $115-124$.

Ziv, Y., 1998. The effect of habitat heterogeneity on species diversity patterns: a community-level approach using an object-oriented landscape simulation model (SHALOM). Ecol. Model. 111, $135-170$.

Zollner, P.A., 2000. Comparing the landscape level perceptual abilities of forest sciurids in fragmented agricultural landscapes. Landsc. Ecol. 15, 523-533.

Zollner, P.A., Lima, S.L., 1999a. Illumination and the perception of remote habitat patches by white-footed mice. Anim. Behav. 58, 489-500.

Zollner, P.A., Lima, S.L., 1999b. Search strategies for landscapelevel interpatch movements. Ecology 80, 1019-1030. 